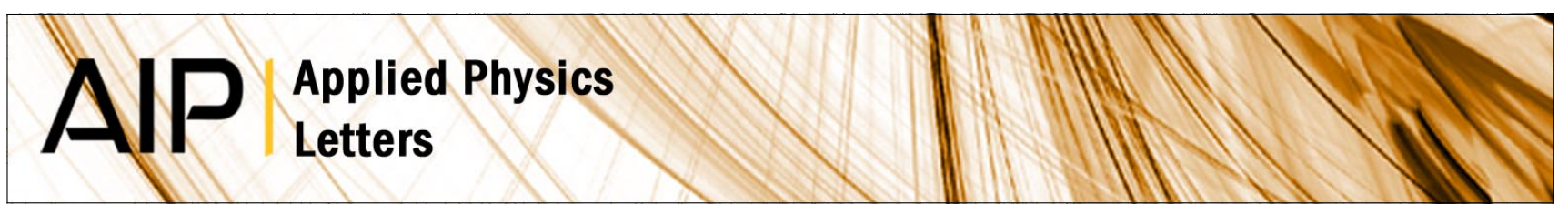

\title{
Anisotropic etching of diamond by molten Ni particles
}

W. Smirnov, J. J. Hees, D. Brink, W. Müller-Sebert, A. Kriele et al.

Citation: Appl. Phys. Lett. 97, 073117 (2010); doi: 10.1063/1.3480602

View online: http://dx.doi.org/10.1063/1.3480602

View Table of Contents: http://apl.aip.org/resource/1/APPLAB/v97/i7

Published by the American Institute of Physics.

\section{Related Articles}

Agglomeration of magnetic nanoparticles

J. Chem. Phys. 136, 124109 (2012)

In-situ and ex-situ characterization of $\mathrm{TiO} 2$ and Au nanoparticle incorporated $\mathrm{TiO} 2$ thin films for optical gas sensing at extreme temperatures

J. Appl. Phys. 111, 064320 (2012)

Tailored assembly of colloidal particles: Alternative fabrication of photonic crystal or photonic glass

Appl. Phys. Lett. 100, 131901 (2012)

Misfit dislocations in multimetallic core-shelled nanoparticles

Appl. Phys. Lett. 100, 111603 (2012)

Explicit all-atom modeling of realistically sized ligand-capped nanocrystals

J. Chem. Phys. 136, 114702 (2012)

\section{Additional information on Appl. Phys. Lett.}

Journal Homepage: http://apl.aip.org/

Journal Information: http://apl.aip.org/about/about_the_journal

Top downloads: http://apl.aip.org/features/most_downloaded

Information for Authors: http://apl.aip.org/authors

\section{ADVERTISEMENT}

\section{(@) ACCELERATE AMBER AND NAMD BY $5 X$. NVIIIA TRYIT ONA FREE, REMOTELYYHOSTED CLUSTER.}




\title{
Anisotropic etching of diamond by molten $\mathrm{Ni}$ particles
}

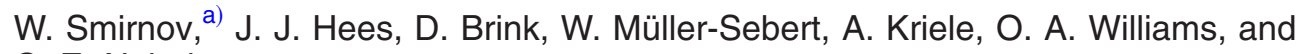 \\ C. E. Nebel \\ Fraunhofer Institut für Angewandte Festkörperphysik, Tullastrasse 72, Freiburg 79108, Germany
}

(Received 31 May 2010; accepted 25 July 2010; published online 19 August 2010)

\begin{abstract}
Nanopores in insulating solid state membranes have recently attracted much interest in the field of probing, characterizing, and manipulating single linear polymers such as DNA/RNA and proteins in their native environment. Here a low cost, fast, and effective way to produce nanostructures such as pyramidal shaped nanopores and nanochannels with dimensions down to about $15 \mathrm{~nm}$ in diamond membranes without any need for electron-beam lithography is demonstrated. By use of a catalytic process, anisotropic etching of diamond with self-organized $\mathrm{Ni}$ nanoparticles in hydrogen atmosphere at $900{ }^{\circ} \mathrm{C}$ is achieved and possible etching mechanisms are discussed. It is shown that diamond planes with the crystallographic orientation of [111] are etched slowest with this method. (C) 2010 American Institute of Physics. [doi:10.1063/1.3480602]
\end{abstract}

Single molecular detection of DNA molecules has been presented by measuring the current blockade of translocating molecules through biased nanopores in a membrane that divides a chamber filled with a conducting electrolyte solution into two compartments. ${ }^{1}$ Each passing molecule blocks an open pore resulting in a reduction in the ionic current. Occurring features in the electrical signal such as time duration and magnitude of current blockage could be used to analyze the characteristics of molecules under investigation. While at the beginning solely natural protein pores were used that can have issues associated with biological structures, later solid state nanopores have been utilized which possess high stability and can withstand pressure variations and mechanical vibrations and thus give more reproducible results. E.g., Storm $e t a l .^{2}$ presented a technique to prepare single-nanometer precision silicon oxide pores by anisotropic etching of singlecrystal $\mathrm{Si}$ in $\mathrm{KOH}$ and a post treatment with high energy electrons (TEM) to shrink the holes in the membrane. The potential of using nanopores has been demonstrated not only to examine biomolecules one by one but also in molecular filter applications. ${ }^{3}$

However, analyzing molecules in harsh conditions such as extreme temperatures, voltages or $p \mathrm{H}$ conditions could result in malfunctioning or in extreme cases to the destruction of sensors with conventional membranes. It is therefore important to establish nanopores in a material that is able to sustain such extremes. Diamond is the perfect candidate for this task since it has exceptional properties such as the highest hardness and Young's modulus (1200 GPa) of all known solids, a very high thermal conductivity $\left(20-22 \mathrm{~W} \mathrm{~cm}^{-1} \mathrm{~K}^{-1}\right)$ and a low thermal expansion coefficient. ${ }^{4-7}$ It can be used to attach binding sites in order to be able to detect specific molecules. ${ }^{8}$ Furthermore due to its wide band gap, it is well known, that the background current of diamond is very low and the potential window very wide compared to conventional electrodes made from metals or graphite. ${ }^{9}$ This behavior enables detection with low noise over a wide potential range. Diamond is chemically inert and as such cannot be etched wet chemically by acids.

\footnotetext{
${ }^{\text {a) }}$ Author to whom correspondence should be addressed. Electronic mail: waldemar.smirnov@iaf.fraunhofer.de.
}

Usually etching of diamond is performed almost exclusively with reactive ions with a resolution that is strongly limited to lithographical processes.

In this letter a low cost, fast, and effective way to etch oriented nanostructures into diamond through the catalytic gasification of carbon by self-assembled nickel nanoparticles generated from a thin Ni layer in hydrogen atmosphere is presented. With this method, slowest etched diamond planes are $\{111\}$ faces, which is comparable to some wet chemical etching experiments of $\mathrm{Si}$ and $\mathrm{Ge}$ whose lattices have also diamond structure. ${ }^{10,11}$ Etch pit phenomena in general are known and it has been reported that etch pit morphologies on a crystallographic face are strongly dependent on various parameters such as concentration of the etchant, the composition, e.g., additive impurities or the temperature. ${ }^{12-15}$ These experiments have shown that the form and morphology of etched crystal faces invariably bear the symmetry of their crystallographic orientations. Owing to this behavior and choosing a diamond crystal with a given orientation, it was possible to etch well oriented pores and channels with a tunable size distribution and control of shape in diamond substrates without any need for lithographic processes.

The etching of the diamond is based on two steps, (i) the formation of self-assembled $\mathrm{Ni}$ nanoparticles on a diamond substrate and (ii) the subsequent use of these particles to etch diamond catalytically. Different types of diamond substrates were used for these experiments, namely microcrystalline diamond (MCD) and single-crystalline diamond (SCD). The MCD was grown in an ellipsoidal shaped microwave plasma enhanced chemical vapor deposition system with following parameters: $900{ }^{\circ} \mathrm{C}$ Temperature, $3000 \mathrm{~W}$ microwave power, $3 \%$ methane in hydrogen, and pressure of 100 mbar. $^{16}$ The MCD films were mechanically polished after growth and had a thickness of about $100 \mu \mathrm{m}$. The crystal grains had a size of about $50 \times 50 \mu \mathrm{m}$. SCD samples were type Ib [111] and IIa [110], Ib [100] oriented crystals purchased from Sumitomo and Element six, respectively.

The particle formation step (i) involves the deposition of thin Ni layers with thicknesses in the range of 1 to $20 \mathrm{~nm}$ by evaporation. These films were then annealed at $1000{ }^{\circ} \mathrm{C}$ in 500 mbar flowing hydrogen with 200 SCCM (SCCM denotes cubic centimeter per minute at STP) for typically $5 \mathrm{~min}$ 


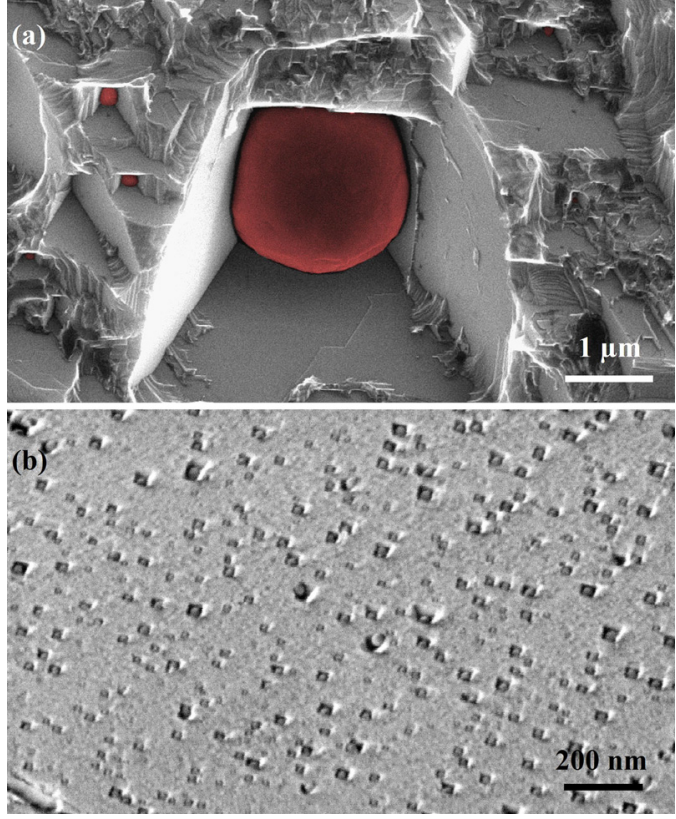

FIG. 1. (Color online) SEM morphologies showing MCD etched by hot Ni spheres. (a) Color enhanced SEM showing etching by big sized Ni particles. (b) Etching diamond by small $(\sim 15 \mathrm{~nm}) \mathrm{Ni}$ particles.

on a graphite plate in an induction furnace. For the etching step (ii) same annealing procedure is carried out as in step (i) but with prolonged annealing time of about 1:30 h.

The base pressure of the furnace before annealing was $<10^{-6}$ mbar. The temperature was increased at a rate of $\sim 300{ }^{\circ} \mathrm{C} / \mathrm{min}$ and measured with a pyrometer with a readings precision of $\pm 5{ }^{\circ} \mathrm{C}$. After the annealing steps, the samples were cooled down for 30 min under continuous hydrogen flow.

During annealing step (i), the Ni layer melts and forms self-organized nanoparticles in order to minimize the surface energy. Ni melting and subsequent formation of nanoparticles occurs at annealing temperatures of around $1000^{\circ} \mathrm{C}$, which is lower than the melting point of bulk Ni $\left(1455^{\circ} \mathrm{C}\right)$. This effect is well known and relates to the melting point lowering effect of thin metal films and hydrogen diffusion into the metal. ${ }^{17-20}$

The diameter of the resulting $\mathrm{Ni}$ particles is estimated to be approximately ten times larger than the initially evaporated Ni layer thickness.

A color enhanced scanning electron microscopy (SEM) image of an etched MCD film by hot Ni particles (red) is shown in Fig. 1(a). It is clearly seen that an anisotropic etching of the diamond has occurred. While the Ni particles are spherically shaped, the etched structures in the diamond substrate have flat sidewall faces and sharply defined edges. From the picture it is obvious, that the size of the etched structures depends on the size of the initially formed Ni particles. Figure 1(b) shows the SEM pictograph of nanoholes in diamond with a lateral size of $15 \pm 5 \mathrm{~nm}$ that were achieved by etching the diamond with $\mathrm{Ni}$ particles that originated from a $1 \mathrm{~nm}$ thin Ni layer. It is evident, that the size of etched structures can be adjusted to a certain extend by the Ni layer thickness.

The etching effect of molten $\mathrm{Ni}$ particles on differently oriented single-crystal diamonds i.e., [100], [110], and [111], is shown in SEM pictographs in Figs. 2(a)-2(c), respectively.

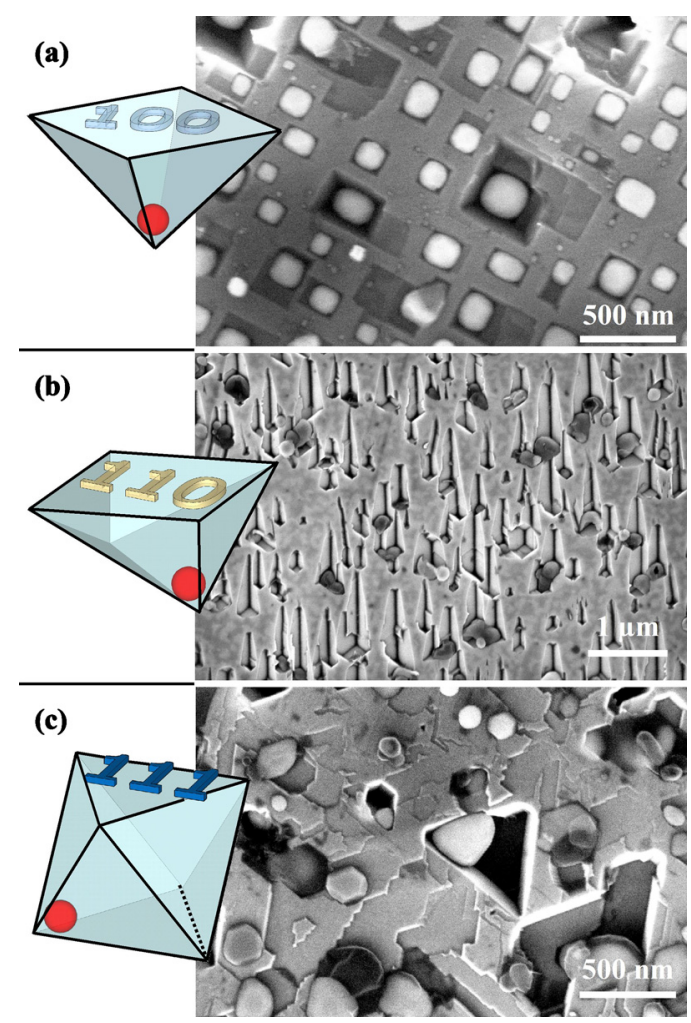

FIG. 2. (Color online) SEM micrograph of observed pitting and channeling of (a) [100], (b) [110], and (c) [111] directed and etched single-crystal diamonds. The inset is a modeled octahedron which reflects symmetries of $\{111\}$ oriented planes with indicated planes. Circles indicate Ni particles (red in online version) (3D animated model enhanced online). [URL: http://dx.doi.org/10.1063/1.3480602.1]

For [100] oriented samples, etch pits with an inverted four sided pyramid are formed. Samples with [110] orientation show elongated etching channels and [111] oriented crystals show etching pits with equilateral triangles and hexagons. These results strongly suggest, that $\mathrm{Ni}$ assisted diamond etching is slowest for [111] oriented diamond planes and that they act as stopping planes.

All eight (111) planes of a cubic cell can be visualized by an octahedron which simply consist of two four sided pyramids connected to each other at their bases. The faces of the octahedron are equilateral triangles. If $\{111\}$ planes are etched slowest, the morphology of the etching pits will reflect the symmetry of this polyhedron. The schemes in Fig. 2 show cross-sections of a modeled octahedron, where all faces are $\{111\}$ planes if not labeled differently. In fact Figs. 2(a) and 2(b) indicate the intersections of the octahedron with $\{100\}$ and $\{110\}$ planes, respectively. In this model red spheres are representative of $\mathrm{Ni}$ particles. It is evident, that the etched structures in the diamond substrates correspond to the modeled pictures. The angle between the edges of the etch pits in the SEM pictograms of Figs. 2(a) and 2(c) are measured to be $90^{\circ}$ and $60^{\circ}$, respectively, which corresponds to the symmetries of the intersected octahedron as expected. If the etching channel for [110] oriented crystals is formed by two $\{111\}$ faces, the enclosed angle will be $\sim 109.47^{\circ}$ as can be obtained by simple vector calculations. To verify this value on the sample, a SEM tilting and length measuring technique was applied: The sample was first rotated, so that the channels were oriented vertically to the screen as is the case in Fig. 2(b). Then the specimen was tilted in small steps 
until one side of the channel was almost perpendicular and the other one was almost not visible since it was parallel to the line of sight. After each tilting step the apparent width (as seen on the screen) of the walls from the channel was measured with help of the scale bar. Plotting and extrapolating the measured lengths against the tilting angles gives an accurate value for the angle that is enclosed by the originally flat surface $\{110\}$ and the inclined channel walls. By this procedure it was possible to determine the enclosed angle $\alpha$ by the two faces forming the channel. The obtained number for $\alpha=110^{\circ} \pm 1^{\circ}$ agrees very well with the theoretical value of $\sim 109.47^{\circ}$.

The analysis of this etching morphologies shows undoubtedly, that the restricting sidewalls of the etching pits and channels are $\{111\}$ planes.

The etching of diamond with $\mathrm{Ni}$ is achieved in a hydrogen gas atmosphere of 500 mbar pressure. The higher the temperature and the higher the pressure of hydrogen gas in the furnace, the faster the diamond is etched. The etching mechanism relates to features which have been characterized in detail for the growth of carbon nanotubes (CNTs). ${ }^{21,22}$ There, Ni particles act as catalysts to decompose methane and to grow carbon filaments with hydrogen exhaust.

For etching diamond with $\mathrm{Ni}$, the model of the process is briefly discussed as follows. It is assumed that hot $\mathrm{Ni}$ particles destroy the crystallinity of diamond at the diamond-Ni interface. $^{23}$ This amorphous carbon might be able to diffuse into and through the $\mathrm{Ni}$ and precipitate at the vacuum interface. $^{24,25}$ Hydrogen, gets catalytically dissociated into hydrogen atoms by the $\mathrm{Ni}$ and gasifies the dissolved carbon in the $\mathrm{Ni}$ and the amorphous carbon at the $\mathrm{Ni}$ interface to form methane or some $\mathrm{CH}_{\mathrm{x}}$ complex. Similar chemical reactions have been detected also on activated carbon and natural graphite crystals as reported by Tomita et al. ${ }^{26,27}$ This group observed the formation of etched channels in graphite substrates which were accompanied by the formation of methane. To achieve nanopores in diamond we suggest to use thinned $\{100\}$ single-crystal diamonds with the presented Nietching technique.

In summary, anisotropic etching of diamond with hot molten Ni particles in hydrogen gas has been shown. Presented experiments reveal that $\mathrm{Ni}$ gives rise to reasonably well defined etching pits in diamond. It is an anisotropic etching where $\{111\}$ lattice planes are etched slowest. The geometrical properties of the etching pits are therefore governed by the size of $\mathrm{Ni}$ particle (diameter) and the relative orientation of the surface with respect to the [111] lattice structure. Etch pits in form of inverted pyramids, channels and triangles were achieved for [100], [110], and [111] oriented single-crystal diamonds, respectively.

The approach demonstrated here provides a simple and promising way toward solid state nanopore next generation DNA sequencing, where especially pyramidal shaped etch pits in diamond are of great interest. Diamond has pronounced benefits compared to other solid state pores due to the extraordinary mechanical and electrochemical properties of diamond.

The authors would like to acknowledge financial support from the Fraunhofer Attract program COMBIO-"Hybrid HF-MEMS Filters for GHz-Communication and capillary MEMS systems for chemical and biochemical Sensing."

${ }^{1}$ J. L. Li, M. Gershow, D. Stein, E. Brandin, and J. A. Golovchenko, Nature Mater. 2, 611 (2003).

${ }^{2}$ A. J. Storm, J. H. Chen, X. S. Ling, H. W. Zandbergen, and C. Dekker, Nature Mater. 2, 537 (2003).

${ }^{3}$ A. Yamaguchi, F. Uejo, T. Yoda, T. Uchida, Y. Tanamura, T. Yamashita, and N. Teramae, Nature Mater. 3, 337 (2004).

${ }^{4}$ B. S. El-Dasher, J. J. Gray, J. W. Tringe, J. Biener, A. V. Hamza, C. Wild, E. Worner, and P. Koidl, Appl. Phys. Lett. 88, 241915 (2006).

${ }^{5} \mathrm{R}$. Berman, The Properties of Diamond (Academic, London, 1979).

${ }^{6}$ O. Auciello, J. Birrell, J. A. Carlisle, J. E. Gerbi, X. C. Xiao, B. Peng, and H. D. Espinosa, J. Phys.: Condens. Matter 16, R539 (2004).

${ }^{7}$ A. Kriele, O. A. Williams, M. Wolfer, D. Brink, W. Muller-Sebert, and C. E. Nebel, Appl. Phys. Lett. 95, 031905 (2009).

${ }^{8}$ S. Q. Lud, M. Steenackers, R. Jordan, P. Bruno, D. M. Gruen, P. Feulner, J. A. Garrido, and M. Stutzmann, J. Am. Chem. Soc. 128, 16884 (2006).

${ }^{9}$ C. E. Nebel, B. Rezek, D. Shin, H. Uetsuka, and N. Yang, J. Phys. D 40, 6443 (2007).

${ }^{10}$ D. B. Lee, J. Appl. Phys. 40, 4569 (1969).

${ }^{11}$ K. E. Bean, IEEE Trans. Electron Devices 25, 1185 (1978).

${ }^{12}$ A. P. Honess, The Nature, Origin, and Interpretation of the Etch Figures on Crystals (Wiley, New York, 1927).

${ }^{13}$ H. E. Buckley, Crystal Growth (Wiley, New York, 1958).

${ }^{14} \mathrm{~K}$. Sangwal, Etching of Crystals: Theory, Experiment, and Application (North-Holland, Amsterdam, 1987).

${ }^{15}$ I. Zubel and M. Kramkowska, Sens. Actuators, A 115, 549 (2004).

${ }^{16}$ M. Füner, C. Wild, and P. Koidl, Appl. Phys. Lett. 72, 1149 (1998).

${ }^{17}$ N. T. Gladkich, R. Niedermayer, and K. Spiegel, Phys. Status Solidi B $\mathbf{1 5}$ 181 (1966).

${ }^{18}$ P. Buffat and J. P. Borel, Phys. Rev. A 13, 2287 (1976).

${ }^{19}$ P. C. Yang, W. Liu, R. Schlesser, C. A. Wolden, R. F. Davis, J. T. Prater, and Z. Sitar, J. Cryst. Growth 187, 81 (1998).

${ }^{20}$ A. Pundt, Adv. Eng. Mater. 6, 11 (2004).

${ }^{21}$ S. Helveg, C. Lopez-Cartes, J. Sehested, P. L. Hansen, B. S. Clausen, J. R Rostrup-Nielsen, F. Abild-Pedersen, and J. K. Norskov, Nature (London) 427, 426 (2004).

${ }^{22}$ L. Ni, K. Kuroda, Z. Ling-Ping, K. Ohta, K. Matsuishi, and J. Nakamura, Carbon 47, 3054 (2009).

${ }^{23}$ R. Anton, Carbon 46, 656 (2008).

${ }^{24}$ J. J. Lander, H. E. Kern, and A. L. Beach, J. Appl. Phys. 23, 1305 (1952).

${ }^{25}$ P. H. Clifton and S. Evans, Ind. Diamond Rev. 55, 26 (1995).

${ }^{26}$ A. Tomita and Y. Tamai, J. Phys. Chem. 78, 2254 (1974).

${ }^{27}$ A. Tomita, N. Sato, and Y. Tamai, Carbon 12, 143 (1974). 